Ferrata Storti Foundation

\title{
Autophagy inhibition as a potential future targeted therapy for ETV6-RUNX1-driven B-cell precursor acute lymphoblastic leukemia
}

Haematologica 2019

Volume 104(4):738-748

\section{Correspondence:}

MONIQUE L. DEN BOER

m.I.denboer@prinsesmaximacentrum.nl

Received: March 18, 2018.

Accepted: October 30, 2018.

Pre-published: October 31, 2018.

doi:10.3324/haematol.2018.193631

Check the online version for the most updated information on this article, online supplements, and information on authorship \& disclosures: www.haematologica.org/content/104/4/738

(C)2019 Ferrata Storti Foundation

Material published in Haematologica is covered by copyright. All rights are reserved to the Ferrata Storti Foundation. Use of published material is allowed under the following terms and conditions:

https://creativecommons.org/licenses/by-nc/4.0/legalcode. Copies of published material are allowed for personal or internal use. Sharing published material for non-commercial purposes is subject to the following conditions:

https://creativecommons.org/licenses/by-nc/4.0/legalcode, sect. 3. Reproducing and sharing published material for commercial purposes is not allowed without permission in writing from the publisher.

\author{
Roel Polak, ${ }^{1}$ Marc B. Bierings, ${ }^{2,3}$ Cindy S. van der Leije, ${ }^{4}$ Mathijs A. Sanders, ${ }^{4}$ \\ Onno Roovers, ${ }^{4}$ João R. M. Marchante, ${ }^{1}$ Judith M. Boer, ${ }^{1}$ Jan J. Cornelissen, ${ }^{4}$ \\ Rob Pieters, ${ }^{3}$ Monique L. den Boer ${ }^{1,3^{*}}$ and Miranda Buitenhuis ${ }^{4 *}$
}

${ }^{1}$ Department of Pediatric Oncology, Erasmus MC - Sophia Children's Hospital, Rotterdam; ${ }^{2}$ Department of Pediatric Oncology, University Medical Center Utrecht; ${ }^{3}$ Princess Máxima Center for Pediatric Oncology, Utrecht and ${ }^{4}$ Department of Hematology, Erasmus Medical Center, Rotterdam, the Netherlands

${ }^{\star} M d B$ and $M B$ contributed equally to this work.

\section{ABSTRACT}

ranslocation $\mathrm{t}(12 ; 21)$, resulting in the ETV6-RUNX1 (or TELAML1) fusion protein, is present in $25 \%$ of pediatric patients with B-cell precursor acute lymphoblastic leukemia and is considered a first hit in leukemogenesis. A targeted therapy approach is not available for children with this subtype of leukemia. To identify the molecular mechanisms underlying ETV6-RUNX1-driven leukemia, we performed gene expression profiling of healthy hematopoietic progenitors in which we ectopically expressed ETV6-RUNX1. We reveal an ETV6-RUNX1-driven transcriptional network that induces proliferation, survival and cellular homeostasis. In addition, Vps34, an important regulator of autophagy, was found to be induced by ETV6-RUNX1 and up-regulated in ETV6-RUNX1-positive leukemic patient cells. We show that induction of Vps34 was transcriptionally regulated by ETV6RUNX1 and correlated with high levels of autophagy. Knockdown of Vps34 in ETV6-RUNX1-positive cell lines severely reduced proliferation and survival. Inhibition of autophagy by hydroxychloroquine, a well-tolerated autophagy inhibitor, reduced cell viability in both ETV6RUNX1-positive cell lines and primary acute lymphoblastic leukemia samples, and selectively sensitized primary ETV6-RUNX1-positive leukemia samples to $\mathrm{L}$ asparaginase. These findings reveal a causal relationship between ETV6-RUNX1 and autophagy, and provide pre-clinical evidence for the efficacy of autophagy inhibitors in ETV6-RUNX1driven leukemia.

\section{Introduction}

Acute lymphoblastic leukemia (ALL) is the most common pediatric malignancy. During the last decades, the overall survival rates of pediatric ALL have improved significantly. ${ }^{1}$ This is primarily due to optimization of conventional chemotherapeutic drug regimens combined with risk-directed therapies. ${ }^{1}$ However, to date, still $20 \%$ of pediatric ALL cases relapse because of resistance to therapy. ${ }^{2}$ In addition, long-term treatment-induced side effects remain considerable. ${ }^{3}$ New treatment regimens increasingly aim to target specific intrinsic characteristics of leukemia. This approach has, for example, led to the successful development of BCR-ABL1 inhibitors. ${ }^{4}$ Regrettably, such a targeted approach is not available for the majority of children suffering from leukemia.

Translocation $\mathrm{t}(12 ; 21)(\mathrm{p} 13 ; \mathrm{q} 22)$, resulting in the ETV6-RUNX1 fusion protein (also known as TEL-AML1), is present in $25 \%$ of pediatric patients with B-cell precursor acute lymphoblastic leukemia (BCP-ALL) and is therefore the most common fusion protein in childhood cancer. ${ }^{5}$ The $\mathrm{t}(12 ; 21)(\mathrm{p} 13 ; \mathrm{q} 22)$ rearrangement fuses the 5' non-DNA binding region of the ETS family transcription factor ETV6 (TEL) to almost the entire RUNX1 (AML1) locus. ${ }^{5,6}$ Despite the favorable prognosis 
associated with this cytogenetic type of BCP-ALL, resistance to chemotherapeutic drugs and relapse occur in approximately $10 \%$ of these patients..$^{7-9}$

The ETV6-RUNX1 fusion protein induces a silent preleukemic clone that requires additional genetic hits for the transition to leukemia. ${ }^{10-12}$ Although these pre-leukemic ETV6-RUNX1-positive hematopoietic stem cells (HSCs) still possess self-renewal properties and are capable of contributing to hematopoiesis, they fail to outcompete normal HSCs. ${ }^{11,12}$ In ETV6-RUNX1-positive leukemia, this early genetic lesion is followed by a number of 'driver' copy number alterations, including loss of ETV6 and alterations directed to genes regulating normal B-cell differentiation. ${ }^{13}$ These alterations are acquired independently without pref- erential order, thereby generating a dynamic clonal architecture. ${ }^{13}$ This genetic variation implies that targeted therapy in ETV6-RUNX1-driven ALL should preferably be directed to targets that are present in all subclones, i.e. those being deregulated by the ETV6-RUNX1 fusion protein itself. This concept is further supported by the observation that ETV6-RUNX1-positive cell lines are highly dependent on the expression of the fusion protein for their survival. ${ }^{14,15}$ Previous reports revealed that enhanced levels of STAT3, heat-shock proteins, survivin, has-mir-125b-2, the erythropoietin receptor, cytoskeleton regulatory genes, and the $\mathrm{PI} K \mathrm{~K} / \mathrm{PKB} / \mathrm{mTOR}$ pathway, as well as aberrant regulation of the TGF $\beta$ pathway, are important for ETV6RUNX1-positive BCP-ALL. ${ }^{15-20}$ However, the molecular
A

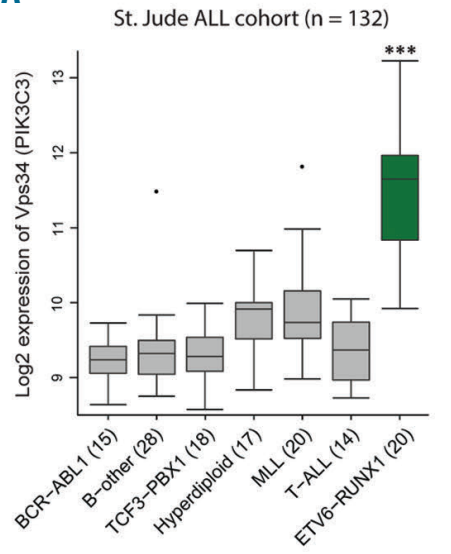

B

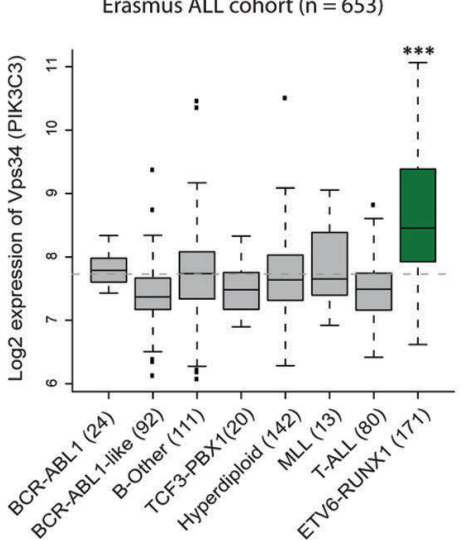

C

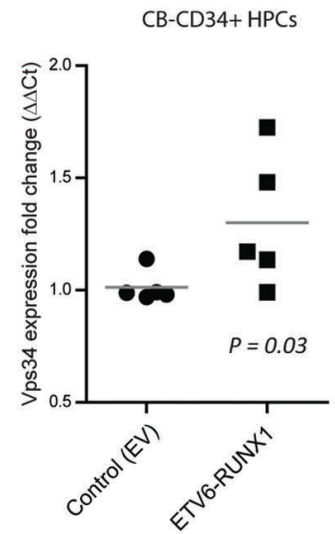

D

SiRNA Knockdown (ETV6-RUNX1+)
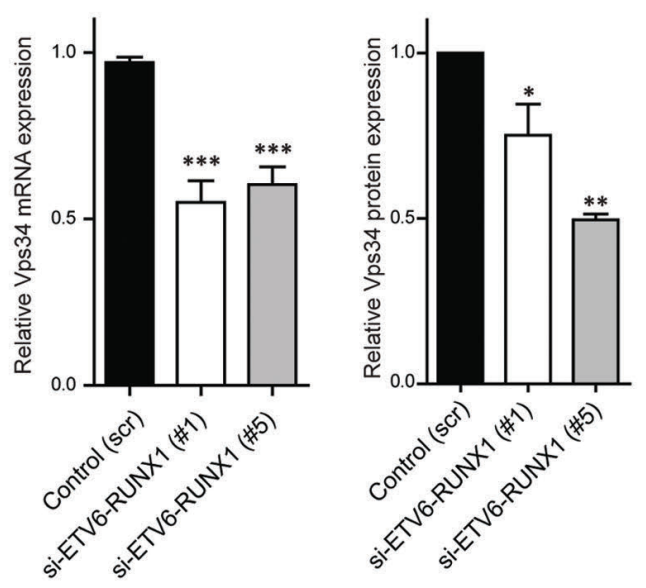

Figure 1. Vps34 is recurrently up-regulated in ETV6-RUNX1-positive B-cell precursor acute lymphoblastic leukemia (BCP-ALL) and is driven by the ETV6-RUNX1 fusion protein. (A) $2 \log$ expression levels of the gene probe set mapped to Vps34 were analyzed in a cohort of 132 pediatric ALL patients published by Yeoh et al. ${ }^{26}$ Gene expression of ETV6-RUNX1-positive patients (green bar) was compared to gene expression of all other B-ALL patients (excluding T-ALL): ***False Discovery Rate (FDR)-adjusted $P=3.45 * 10^{-15}$. (B) In addition, $2 \log$ expression levels of the gene probe set mapped to Vps34 were analyzed in a cohort of 653 pediatric ALL patients published by Van der Veer et al. ${ }^{30}$ Gene expression of ETV6-RUNX1-positive patients (green bar) was compared to gene expression of all other B-ALL patients (excluding T-ALL). Gray dashed line represents mean expression of all patients: $* * *$ FDR-adjusted $P=7.24 * 10^{-39}$. (C) CB-CD34 ${ }^{+}$cells were transduced with ETV6RUNX1-IRES-eGFP or with control EV-IRES-eGFP after which eGFP+ cells were sorted and Vps34 mRNA levels were determined by Q-PCR and normalized to hypoxanthine-guanine phosphoribosyltransferase (HPRT). Gray bars represent the mean of 5 biological replicates. Gene expression of Vps34 was compared between ETV6RUNX1+ $1^{+} B-C D 34^{+}$cells and EV-control CB-CD34+ cells ( $n=5 ; P=0.03$ ). (D) ETV6-RUNX1-positive cells were transfected with siRNAs directed to the ETV6-RUNX1 breakpoint or scrambled control siRNAs. Vps34 mRNA levels were determined in ETV6-RUNX1 ${ }^{+}$cells (REH ( $\left.n=2\right)$ and ETV6-RUNX1 transduced CB-CD34 ${ }^{+}$cells ( $\left.n=2\right)$ by QPCR, normalized to HPRT, and compared to the average expression of cells transfected with scrambled control siRNAs ( $n=3 ; P \leq 0.001$ ). Vps34 protein expression was quantified in REH cells by western blot and compared to protein expression of REH cells transfected with scrambled control siRNAs ( $n=2 ; P \leq 0.05$ and $P \leq 0.01$ for siRNA\#1 and siRNA\#5, respectively). Bars represent mean. Error bars represent Standard Error of Mean. $* P \leq 0.05, * * P \leq 0.01$, $* * * P \leq 0.001$. See also Online Supplementary Figures S1-S5. 
A
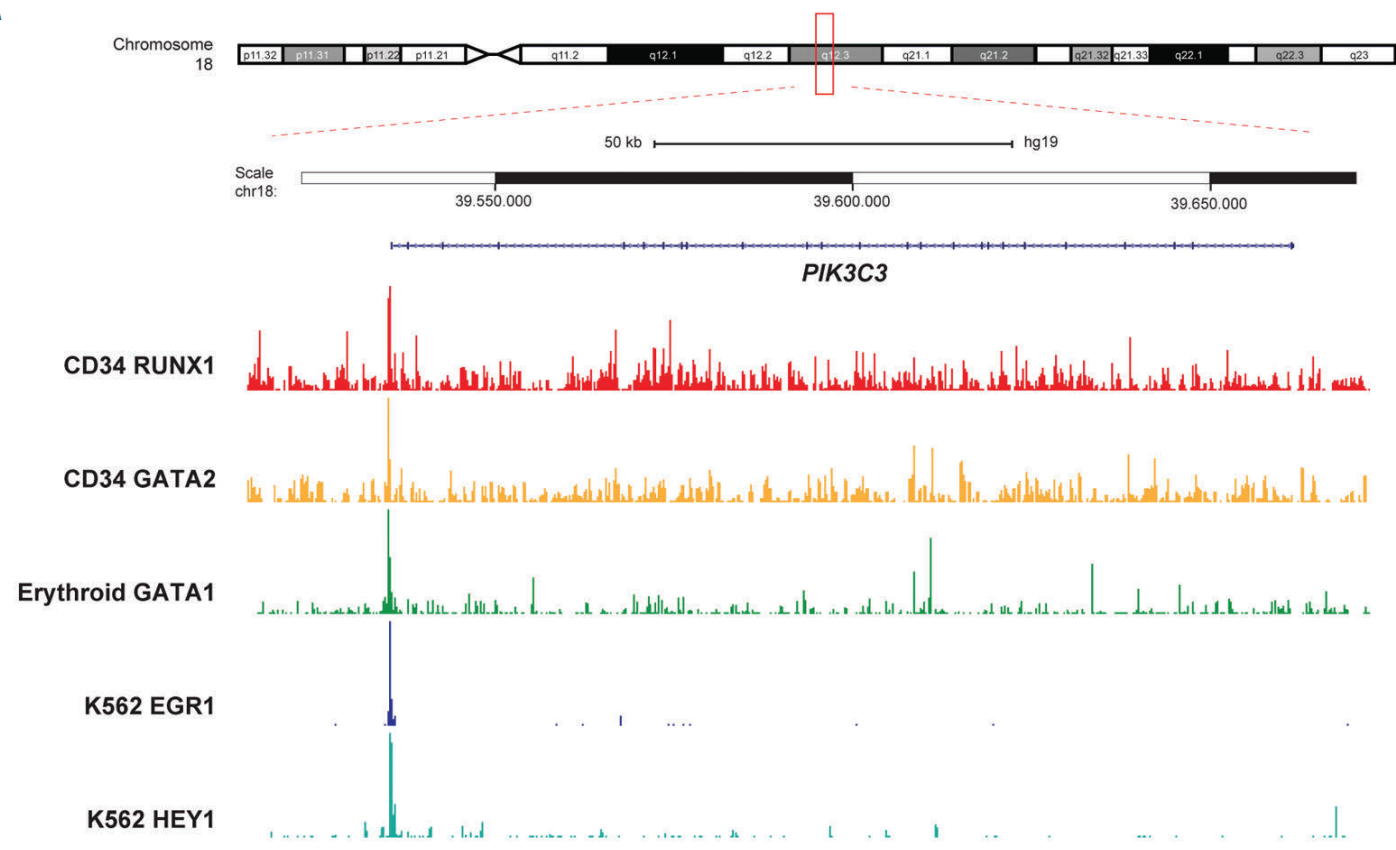

B

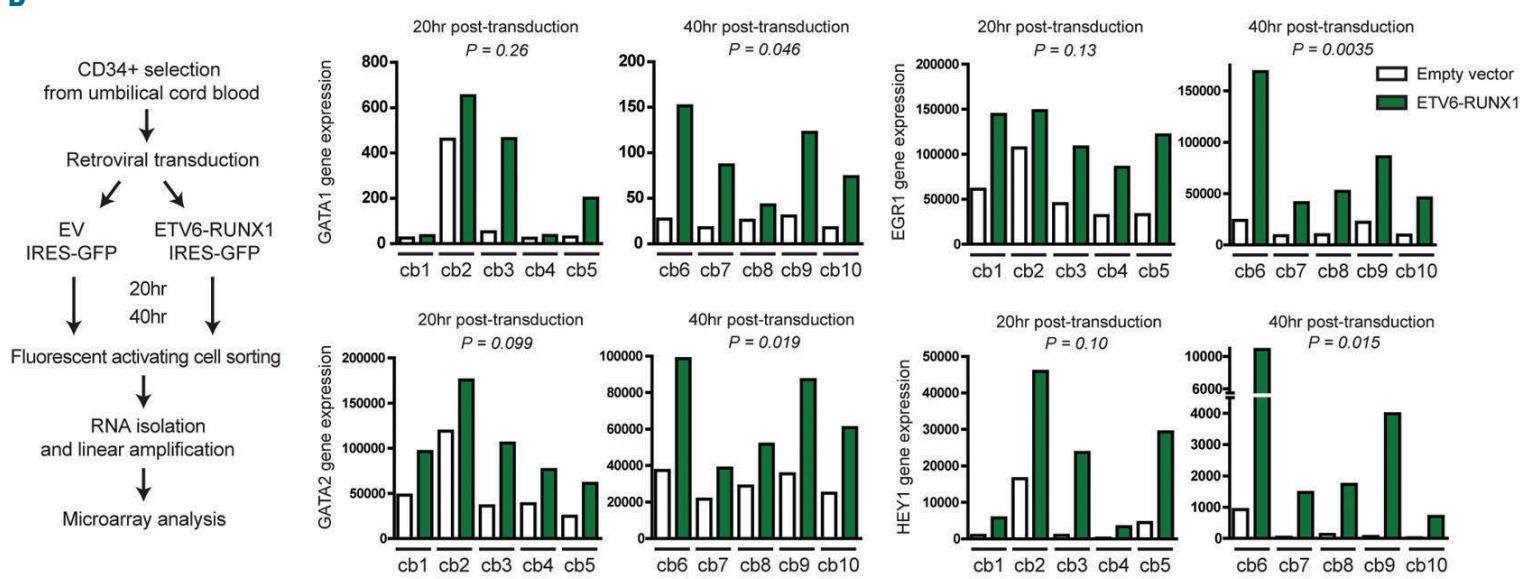

C
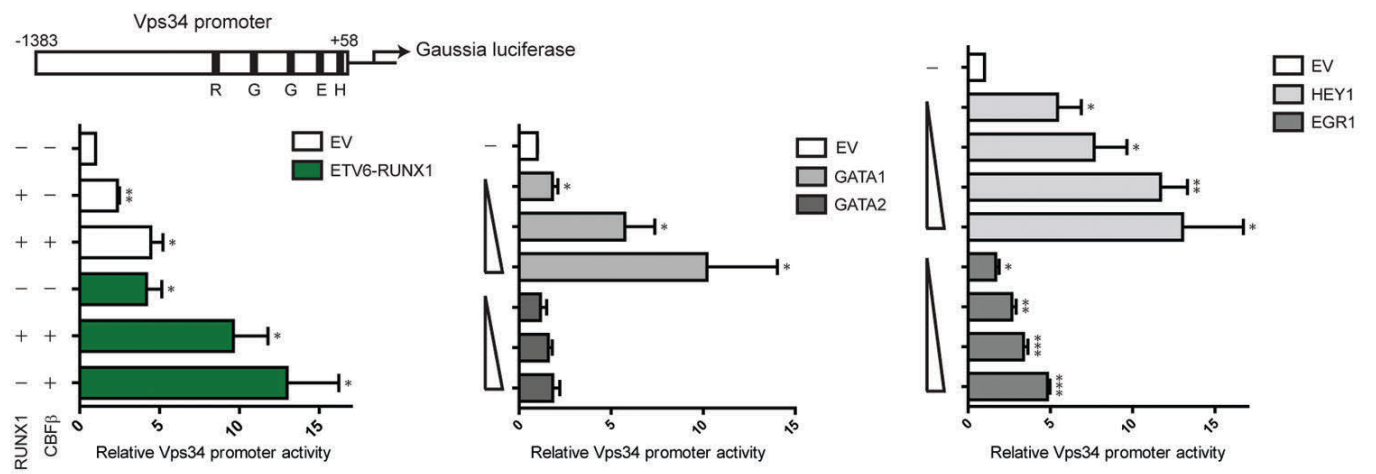

Figure 2. ETV6-RUNX1 and ETV6-RUNX1 target genes enhance Vps34 promoter activity. (A) The UCSC genome browser (GRCh37/hg19) was used to analyze the transcription factors that bind to the Vps34 promoter. Several transcription factors known to play an important role in regulation of hematopoiesis were found to be interacting with this promoter region. Using publically available ChIP-seq data, the interaction of the transcription factors RUNX1, GATA1, GATA2, EGR1 and HEY1 with the Vps34 promoter is shown. (B) Umbilical cord blood-derived CD34+ cells were retrovirally transduced to ectopically express the ETV6-RUNX1 fusion protein. Gene expression analysis was performed 20 and 40 hours (hr) after transduction. For flow chart; see left panel. The gene expression levels of GATA1, GATA2, EGR1 and HEY1 are shown. P-values represent the differences between ETV6-RUNX1 positive (green bars) compared to ETV6-RUNX1-negative (white bars) CB-CD34 ${ }^{+}$cells (FDR-adjusted). (C) (Top) Schematic representation of the -1383 to +58 region of the Vps34 promoter cloned upstream of the Gaussia luciferase gene. Bar graphs: HEK293T cells were co-transfected with a Vps34 promoter construct, RUNX1 and/or CBF $\beta$ (left), increasing concentrations of GATA1 and GATA2 (middle) or HEY1 and EGR1 (right) expression constructs. Luciferase activity was determined $48 \mathrm{hr}$ after transfection. eGFP expression was quantified in each sample by flow cytometric analysis and used to normalize luciferase activity. Data were depicted as fold induction compared to empty vector controls. Error bars represent Standard Error of Mean. ${ }^{*} P \leq 0.05, * * P \leq 0.01, * * * P \leq 0.001$. See also Online Supplementary Figure $S 2 A-C$. 
network underlying the persistence and maintenance of ETV6-RUNX1 BCP-ALL remains to be elucidated.

In the present study, we address the role of autophagy in ETV6-RUNX1-driven leukemia. Autophagy is a cellular recycling system in which unwanted or damaged cellular components are degraded and recycled. The core autophagy-regulating complex includes Vps34, Beclin-1, and Vps15.,21, Although autophagy can sustain cell survival during stress conditions, it can also result in cell death because of progressive cellular consumption. ${ }^{23}$ Whether autophagy plays an initiating or suppressive role in cancer is a question of debate and most likely depends on the (onco)genetic context of cells. ${ }^{24,25}$ This potential dual role of autophagy in cancer highlights the importance of studies on the context-specific role and the functional importance of autophagy in neoplastic processes before the start of autophagy-based therapeutic interventions. We show here that ETV6-RUNX1 targets the autophagy process, which in turn affects sensitivity to LAsparaginase, a key enzyme used in the treatment of ALL that affects the asparagine (and to a lesser extent glutamine) levels in cells.

\section{Methods}

Transduction and gene expression profiling of primary cells

CD34-positive hematopoietic progenitor cells (CB-CD34+ cells) were derived from human cord blood and transduced with retrovirus expressing ETV6-RUNX1 and eGFP. DAPI-CD34+ $\mathrm{GFP}^{+} \mathrm{CB}-$ CD34+ cells were sorted using a BD ARIA II sorter. After sorting, cells were lysed and RNA was extracted and subsequently linearly amplified.

Bone marrow aspirates were obtained from children with newly diagnosed BCP-ALL prior to treatment. Leukemic blasts were collected and processed as previously described.

Affymetrix GeneChip HG-U133-Plus-2.0 microarrays were used for all samples. Microarray data of $\mathrm{CB}-\mathrm{CD} 34^{+}$cells are available in the ArrayExpress database under accession number $\mathrm{E}$
A

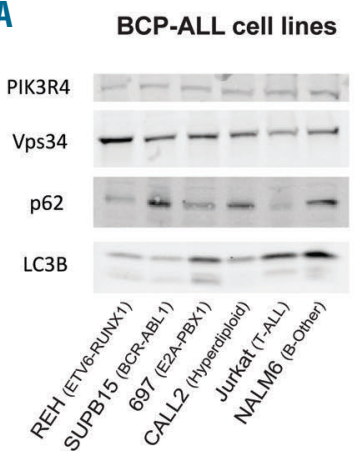

B

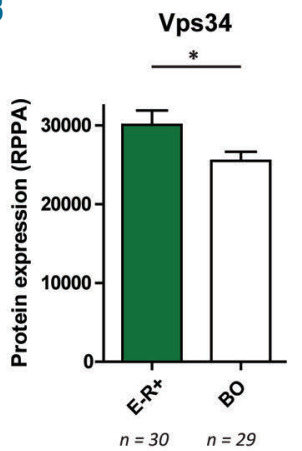

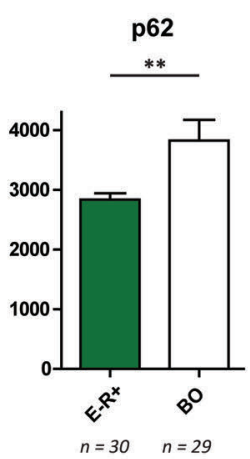

C

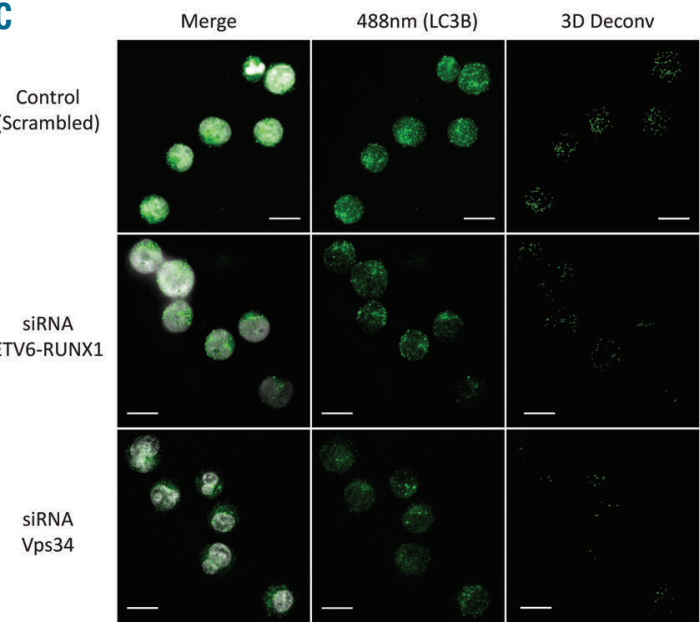

D LC3B+ vesicles

$\mathrm{E}$
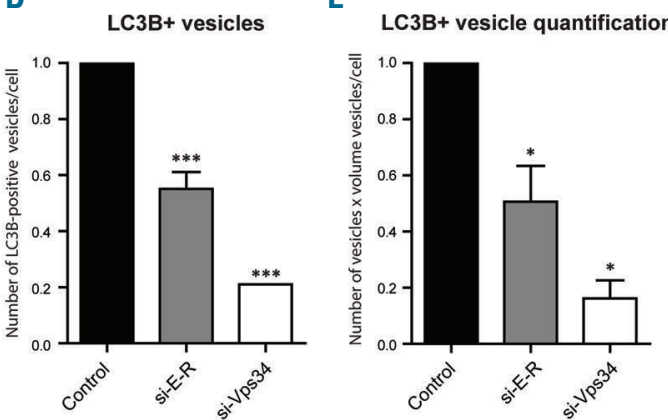

Figure 3. Autophagy levels are high in ETV6-RUNX1-positive B-cell precursor acute lymphoblastic leukemia (BCP-ALL) and regulated by ETV6-RUNX1 and Vps34. (A) Western blot analysis was performed to determine the expression levels Vps34, p62 (sequestosome 1), and LC3B in ALL cell lines. PIK3R4 was used as a loading control. (B) Quantification of protein levels of Vps34 and p62 measured by reverse phase protein array (RPPA) in 30 ETV6-RUNX1-positive primary BCP-ALL patient samples (ETV6-RUNX1+), and 29 B-Other primary BCP-ALL patient samples (BO). Data are means \pm Standard Error of Mean. * $P \leq 0.05, * * P \leq 0.01$. (C) Representative confocal images showing LC3B-positive vesicles in ETV6-RUNX1-positive BCP-ALL cell line REH. (Left) Overlay of LC3B expression and DAPI staining (nuclear staining). (Middle) Only LC3B expression. (Right) 3D representations after deconvolution of the 488nm signal representing the LC3B expression. For the quantification of number and volume of LC3B-positive vesicles, we excluded cells with atypical nuclei. (Top) Control conditions after transfection with scrambled siRNAs. Bottom 6 panels represent conditions after transfection with siRNAs against ETV6-RUNX1 or Vps34. (D and E) Quantification of the number of LC3B-positive vesicles after 3D deconvolution of images (D), and quantification of the number of LC3B-positive vesicles multiplied by the volume of these vesicles after 3D deconvolution of images $(E)(n=3, * P \leq 0.05, * * * P \leq 0.001)$. See also Online Supplementary Figure S6. 
MTAB-3466. Microarray data of BCP-ALL blasts are available in the Gene Expression Omnibus database.

Informed consent was provided according to the Declaration of Helsinki. Use of left-over materials for research purposes was approved by the Institutional Review Board (IRB) of the University Medical Center Rotterdam; IRB approval file number MEC 2004-203.

\section{Functional assays}

For protein quantification, both western blot analysis and reverse phase protein arrays (RPPA) were used. Vps34 promoter activity was studied using a reporter construct consisting of a $1.4 \mathrm{kB}$ region of the Vps34 promoter upstream of the Gaussia luciferase gene. Cell viability was quantified using MTT cytotoxicity assays or flow cytometry-based Annexin V - Propidium Iodide assays. Silencing of genes was achieved using transfec- tion of specific siRNAs or a lentiviral knockdown approach using the $\mathrm{pLKO} .1$ Mission vector containing a puromycin selection marker. Autophagy levels (number and volume of LC3Bpositive vesicles) were quantified using confocal scanning microscopy (Leica SP5).

\section{Statistical analysis}

Statistical analysis of microarray data of paired CB-CD $34^{+}$cells was performed using a linear mixed model. Microarray data of primary ALL samples were analyzed using LIMMA. Functional analysis of differential gene expression was performed using OIAGEN's Ingenuity Pathway Analysis. Both the Student $t$-test and the Student paired $t$-test were used when applicable. Bar graphs represent the mean of biological replicates. Error bars represent standard error of mean (SEM). Further details of the methods used are available in the Online Supplementary Appendix.
A

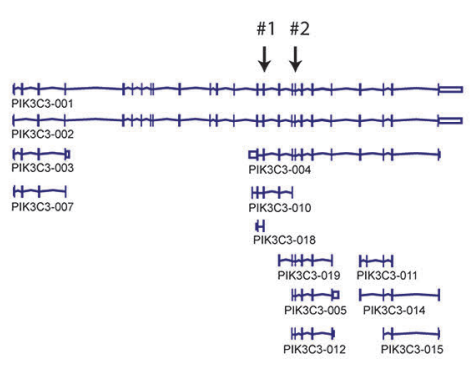

B

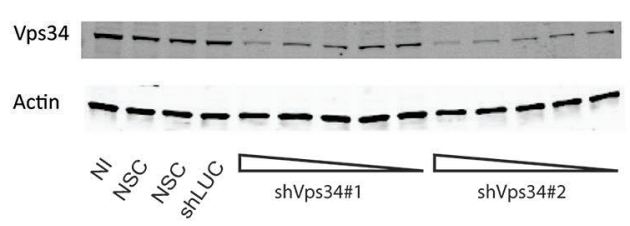

D

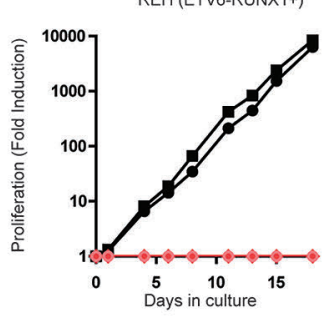

Active cell cycle day 7

$\mathrm{E}$

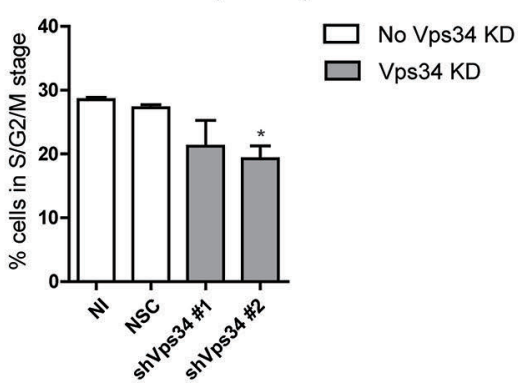

REHS1 (ETV6-RUNX1+)

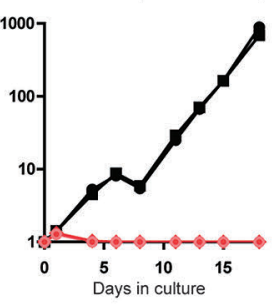

$\mathrm{F}$

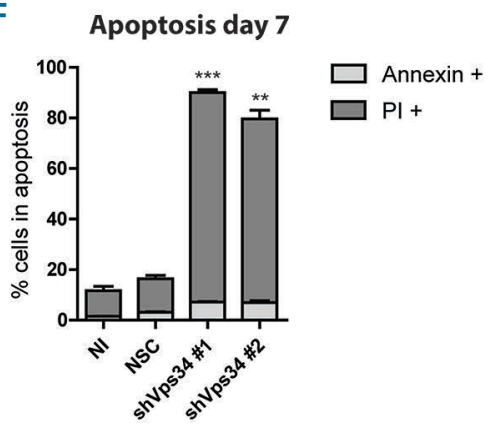

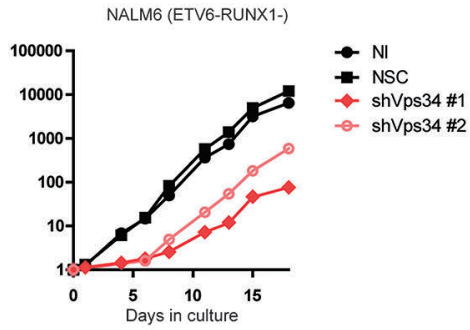

C

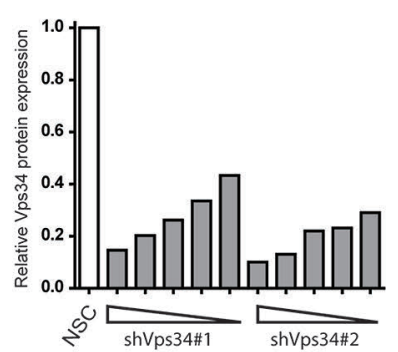

shVps34\#1 shVps34\#2

Figure 4. Vps34 is essential for the survival of ETV6-RUNX1-positive leukemic cells. (A) Schematic representation of the known transcript variants of Vps34 (Ensemble Genome Browser; ENSG00000078142) and the shVps34\#1 and shVps34\#2 recognition sites. (B) ETV6-RUNX1-positive (REH) BCP-ALL cells were lentivirally transduced with scrambled shRNA control, or two distinct shRNA constructs to silence Vps34 expression. Western blot analysis was performed with an antibody against Vps34 or $\beta$-actin to visualize the knockdown of Vps34 in REH cells. A representative experiment is shown in which increasing concentrations of virus were used to emphasize the specificity of Vps34 knockdown. (C) Data were quantified and are depicted as the relative Vps34 expression in comparison to the expression in cells transduced with scrambled (non-silencing) controls. (D) ETV6-RUNX1-positive (REH and REHS1) and ETV6-RUNX1-negative (NALM6) BCP-ALL cells were lentivirally transduced with scrambled shRNA control (NSC) or two distinct Vps34 shRNA constructs. NI: non-infected cells. Cells were cultured for 18 days. To determine the effect on proliferation, cell counts were performed every 2-3 days. Representative graphs are shown ( $n=3)$. ( $E$ and F) ETV6-RUNX1-positive (REH) BCP-ALL cells were lentivirally transduced with scrambled shRNA control (NSC) or two distinct shRNA constructs to silence Vps34 expression. After seven days of culture, flow cytometrical analysis was performed to determine the effect of Vps34 knockdown on survival and cell-cycle progression. (E) The percentage of viable (AnnexinV-positive, Propidium-lodide negative), actively cycling cells was determined using DyeCycle. Data are shown as the percentage of cells in S, G2 M phase ( $\mathrm{n}=2 ; * P \leq 0.05)$. Error bars represent Standard Error of Mean (SEM). (F) The percentages of early apoptotic (AnnexinV-positive, Propidium-lodide negative) and late apoptotic (Propidium-lodide positive) cells were determined seven days after transduction $(n=2, * * P \leq 0.01, * * * P \leq 0.001)$. Error bars represent SEM. See also Online Supplementary Figure S7. 


\section{Results}

Vps34 is up-regulated in ETV6-RUNX1-positive BCP-ALL and is induced by the ETV6-RUNX1 fusion protein

To identify novel ETV6-RUNX1 target genes, we compared gene expression profiles of primary ALL samples with those of ETV6-RUNX1-transduced umbilical cord blood-derived, healthy CD34-positive hematopoietic progenitors (CB-CD34+) (Online Supplementary Figure S1A). Gene expression analysis revealed that 196 genes were differentially expressed in ETV6-RUNX1 transduced CB CD34+ cells in comparison to empty vector controls (2-40 fold; $P \leq 0.05$ ) (Online Supplementary Figure $S 1 B$ ). Ingenuity Pathway Analysis on these 196 genes predicted an interacting gene network of 36 genes (Online Supplementary Figure S1C). Analysis of gene ontology (GO) functional categories indicated that ETV6-RUNX1 induced a signature associated with pro-survival and pro-proliferative gene expression and cellular homeostasis (Online
Supplementary Figure S1D and E and Online Supplementary Figure S2A and B).

For the gene expression analysis of primary ALL samples, we used data from cohorts in which ETV6 RUNX1positive BCP-ALL patients were included $\mathrm{d}^{26-30}$ and data from an ETV6-RUNX1 knockdown study performed in a leukemic cell line ${ }^{31}$ (Online Supplementary Figure S3). Pathway analysis on the top 500 differentially expressed genes in the largest patient cohort (Erasmus MC cohort, 654 ALL patients including 172 ETV6-RUNX1-positive BCP-ALL patients and 401 ETV6vRUNX1-negative BCP ALL patients ${ }^{30}$ ) revealed a pro-survival and pro-proliferative signature in ETV6 RUNX1-positive patient cells, similar to the phenotype predicted in CB-CD34 cells ectopically expressing ETV6-RUNX1 (Online Supplementary Table S1 and Online Supplementary Figure S2C).

The class III PI3 kinase Vps34 (PIK3C3) was found to be recurrently up-regulated in ETV6-RUNX1-positive ALL patient cells (2.7 fold-higher expression in Erasmus MC
A

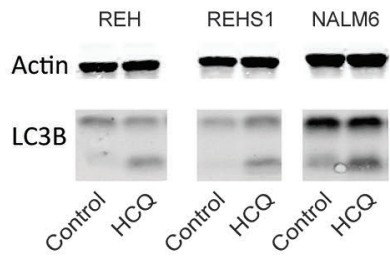

B

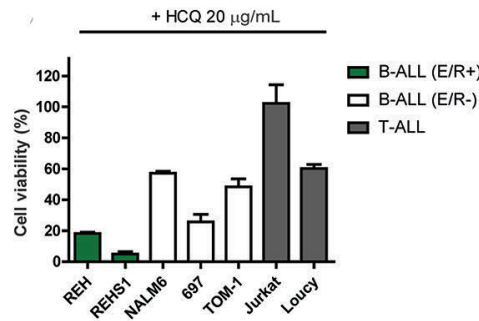

C

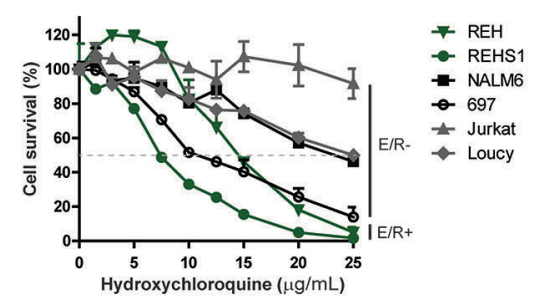

D No HCQ Primary ETV6-RUNX1 positive BCP-ALL
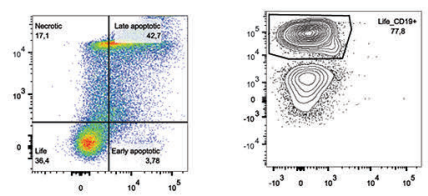

$28.3 \%$

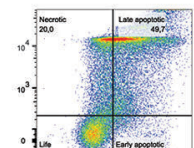

$5 \mu \mathrm{g} / \mathrm{mL}$

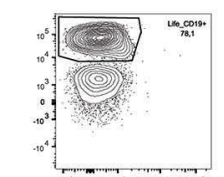

$20.5 \%$
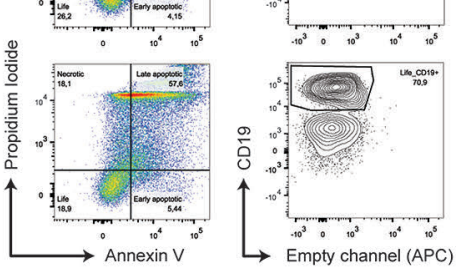

Primary ETV6-RUNX1-positive BCP-ALL

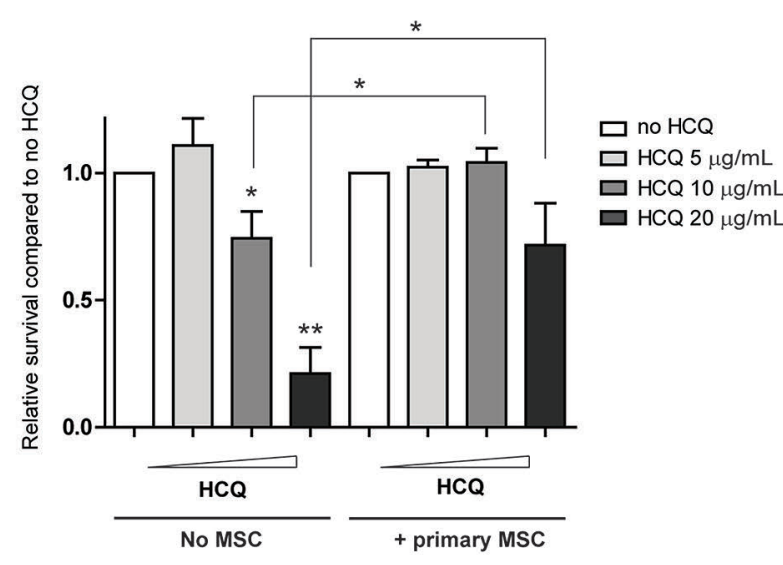

Figure 5. ETV6-RUNX1-positive acute lymphoblastic leukemia (ALL) cells are relatively sensitive to treatment with hydroxychloroguine (HCQ). (A) ETV6-RUNX1-positive (REH and REHS1) and ETV6-RUNX1-negative (NALM6) BCP-ALL cells were cultured in absence or presence of HCQ $(6.25 \mu \mathrm{g} / \mathrm{mL}$ ) for 48 hours. Western blot analysis was performed using an antibody against LC3B to determine the effectiveness of HCQ treatment. LC3B-I represents the cytosolic form of LC3B, while LC3BII represents the autophagosome membrane-bound form of LC3B (see also Figure 7). HCQ treatment leads to the accumulation of autophagosomes by blocking the fusion of autophagosomes to lysosomes and hence to an increase in LC3B-II in cells. (B) ETV6-RUNX1-positive BCP-ALL, ETV6-RUNX1-negative BCP-ALL, and T-ALL cell lines were cultured in absence or presence of $\mathrm{HCQ}(20 \mathrm{\mu g} / \mathrm{mL})$ for four days. An MTT assay was performed to determine the effect of HCQ treatment on the viability of the cells. Data are depicted as the percentage of viable cells compared to untreated control. Error bars represent Standard Error of Mean (SEM) ( $n=3)$. (C) ETV6-RUNX1-positive BCP-ALL, ETV6-RUNX1-negative BCP-ALL, and T-ALL cell lines were cultured in absence or presence of increasing concentrations of HCQ for four days. An MTT assay was performed to determine the effect of HCQ treatment on the viability of the cells. A representative experiment is shown in which data are depicted as the percentage of viable cells. (D) Primary ETV6-RUNX1-positive BCP-ALL cells were cultured in absence or presence of 5 or $10 \mu \mathrm{g} / \mathrm{mL}$ HCQ for five days. Flow cytometric analysis was performed to determine the percentage of non-apoptotic (Annexin-V negative, Propidium-lodide negative, CD19-positive) cells. Representative FACS plots are shown $(n=5)$. (E) Co-culture experiments were performed with primary ETV6-RUNX1-positive BCP-ALL cells and mesenchymal stromal cells (MSCs). Cells were cultured in absence or presence of increasing concentrations of HCQ for five days. Flow cytometrical analysis was performed to determine the percentage of non-apoptotic (Annexin V-negative, Propidium lodide-negative, CD19-positive) cells. Data are depicted as the relative reduction in survival com-

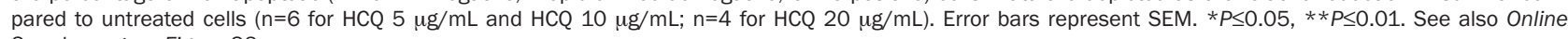
Supplementary Figure S8. 
cohort, FDR-adjusted P=7.24E-39) (Figure $1 \mathrm{~A}$ and B and Online Supplementary Figure S3). To determine the direct effect of the ETV6-RUNX1 fusion protein on Vps34 expression, ETV6-RUNX1 was ectopically expressed in CB-CD34+ cells. Forty hours after transduction, Vps34 mRNA expression was significantly up-regulated by 1.3 fold $(P=0.03)$ (Figure 1D), suggesting a causal relationship between ETV6-RUNX1 and Vps34. Reciprocal experiments were performed in ETV6-RUNX1-positive cells (REH cell line and ETV6-RUNX1 transduced CB-CD34+ cells) using siRNAs directed to the ETV6-RUNX1 breakpoint. Although the ETV6-RUNX1 mRNA levels could only be reduced by $30-35 \%(P \leq 0.05)$ (Online Supplementary Figure S5B), this reduction was sufficient to significantly reduce the levels of Vps34 mRNA and protein both by approximately $40 \%(P \leq 0.05)$ (Figure 1D).

\section{ETV6-RUNX1 and ETV6-RUNX1 target genes enhance Vps34 promoter activity}

The upregulation of Vps34 expression in ETV6-RUNX1positive BCP-ALL patients and ETV6-RUNX1 transduced CB-CD34+ cells, suggests that the Vps34 promoter is activated directly or indirectly by ETV6-RUNX1. Analysis of the Vps34 promoter, using publically available ChIP-seq data, revealed that transcription factors known to play an important role in regulation of hematopoiesis, including
GATA1, GATA2, EGR1 and HEY1, can interact with the Vps34 promoter (Figure 2A). Four of these transcription factors, namely GATA1, GATA2, EGR1 and HEY1, were also found to be up-regulated in ETV6-RUNX1-transduced CB-CD34 $4^{+}$cells (Figure 2B and Online Supplementary Figure $S 1 C$ and Online Supplementary Figure S4). The mRNA expression levels of these four genes were modestly increased in ETV6-RUNX1-transduced CBCD34+ cells 20 hours after transduction and significantly up-regulated after 40 hours: GATA1, GATA2, EGR1, and HEY1 were up-regulated 3.8-fold $(P=0.046), 2.2$-fold $(P=0.019)$, 5.0-fold $(P=0.004)$, and 24.9-fold $(P=0.015)$, respectively (Figure $2 \mathrm{~B}$ ).

To investigate the role of ETV6-RUNX1 and the transcription factors GATA1, GATA2, EGR1, and HEY1 in regulation of the activity of the Vps34 promoter, luciferase reporter assays were performed using a reporter construct consisting of the Gaussia luciferase gene downstream of a $1.4 \mathrm{kB}$ region (-1383 to +58 ) of the Vps34 promoter (Figure 2C). Although RUNX1 expression alone was sufficient to induce Vps34 promoter activity (2.4-fold compared to control, $P \leq 0.01$ ), co-expression of its co-factor $\mathrm{CBF} \beta$ further enhanced Vps34 promoter activity (4.5-fold compared to control, $P \leq 0.05$ ). Similarly, expression of ETV6RUNX1 was sufficient to induce Vps34 promoter activity (4.2-fold compared to control, $P \leq 0.05$ ), and luciferase

A + L-Asparaginase

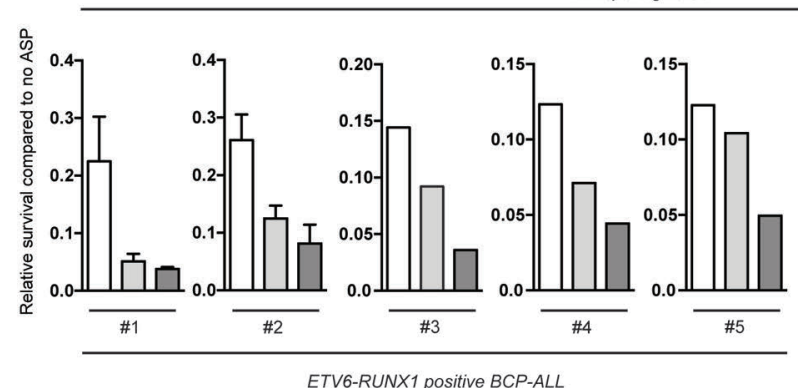

B

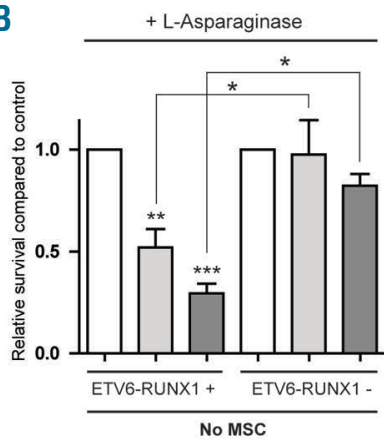

C

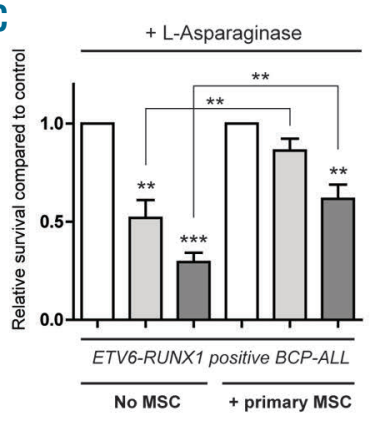

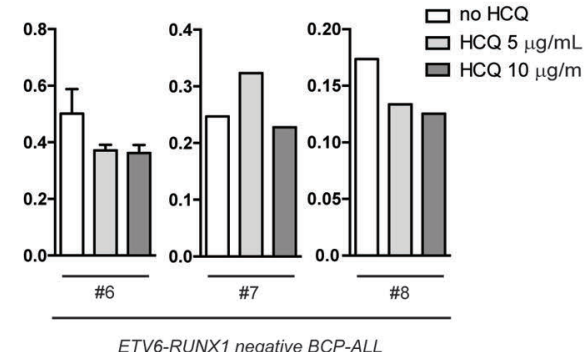

D

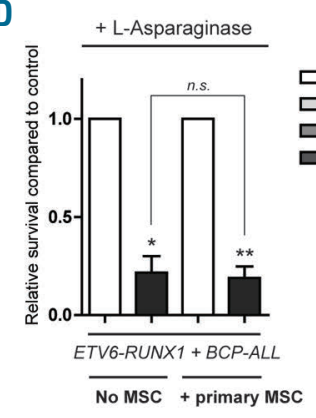

Figure 6. Autophagy inhibition sensitizes ETV6-RUNX1-positive acute lymphoblastic leukemia (ALL) cells to L-Asparaginase. (A) Primary ETV6-RUNX1-positive BCPALL cells were cultured in absence or presence of IC 50 concentrations of L-Asparaginase and increasing concentrations of hydroxychloroquine (HCQ). Flow cytometric analysis was performed to determine the percentage of non-apoptotic (Annexin V-negative, Propidium lodide-negative, CD19-positive) cells (for gating strategy see also Online Supplementary Figure S7C). The survival of primary leukemic blasts in the presence of L-Asparaginase was compared to their survival in the absence of L-Asparaginase. White bars represent the relative survival in the absence of HCQ. Gray bars represent the relative survival in the presence of HCQ: $5 \mu \mathrm{g} / \mathrm{mL} \mathrm{HCQ}$ (light gray) and $10 \mu \mathrm{g} / \mathrm{mL} \mathrm{HCQ}$ (gray). (B) Averages of data presented in (A), representing sensitization of primary leukemic blasts by HCQ to L-Asparaginase ( $\mathrm{n}=5$ for ETV6-RUNX1-positive; $\mathrm{n}=3$ for ETV6-RUNX1-negative primary patient cells; $* P \leq 0.05, * * P \leq 0.01, * * * P \leq 0.001$ ). Error bars represent Standard Error of Mean (SEM). ( $C$ and $D)$ Co-culture experiments were performed with primary ETV6-RUNX1-positive BCP-ALL cells and mesenchymal stem cells (MSC). Cells were cultured in the presence or the absence of L-Asparaginase and increasing concentrations of HCQ. Flow cytometric analysis was performed to determine the percentage of nonapoptotic (Annexin V-negative, Propidium-lodide-negative, CD19-positive) cells. First, the survival of primary leukemic blasts in the presence of L-Asparaginase was compared to their survival in absence of L-Asparaginase. Next, data are depicted as fold reduction compared to HCQ-untreated controls ( $n=5$ for conditions in the absence of MSCs, $n=7$ for conditions in the presence of MSCs for (C), n=3 for conditions in the absence of MSCs, $n=4$ for conditions in the presence of MSCs for (D). ${ }^{*} P \leq 0.05, * * P \leq 0.01, * * * P \leq 0.001$. Error bars represent SEM. See also Online Supplementary Figure S9. 
expression was further enhanced by co-expression of $\mathrm{CBF} \beta$ (13-fold compared to control, $P \leq 0.05$ ). These results demonstrate that, although both RUNX1 and ETV6RUNX1 function as transcriptional activators of Vps34, ETV6-RUNX1 induces Vps34 promoter activity more efficiently (Figure $2 \mathrm{C}$ ). In addition, these results demonstrate that $\mathrm{CBF} \beta$ acts as a co-activator for ETV6-RUNX1 in inducing Vps34 promoter activity. Additional luciferase reporter assays revealed that the ETV6-RUNX1 target genes HEY1, EGR1 and GATA1 induce Vps34 promoter activity in a dose-dependent manner. HEY1, EGR1, and GATA1 induced Vps34 promoter activity up to 13-fold ( $P \leq 0.05)$, 5.0-fold ( $P \leq 0.001)$, and 10-fold $(P \leq 0.05)$, respectively (Figure 2C). GATA2 expression did not induce luciferase expression, which was in concordance with the absence of a GATA2 DNA binding domain in the $-1338 /+58$ promoter region used for the reported assays (Figure $2 \mathrm{~A}$ and $\mathrm{C}$ ). Together, these results demonstrate that the Vps34 promoter is not only positively regulated by the ETV6-RUNX1 fusion protein itself, but also by its target genes HEY1, EGR1 and GATA1.

\section{Autophagy levels are high in ETV6-RUNX1-positive BCP-ALL and regulated by ETV6-RUNX1 and Vps34}

Since Vps34 is a key player in autophagy regulation, we hypothesized that ETV6-RUNX1-mediated upregulation of Vps34 induces autophagy in BCP-ALL cells. To investi- gate this, autophagy levels were determined in ALL cell lines and primary BCP-ALL samples by western blot analysis and RPPA. Western blot analysis in a panel of ALL cell lines revealed that Vps34 protein levels are the highest in the ETV6-RUNX1-positive cell line REH (Figure 3A). In comparison to ETV6-RUNX1-negative ALL cell lines, lower levels of p62 (SOSTM1) and LC3B, both specifically degraded by autophagy, were observed in REH cells. These results suggest high levels of autophagy in REH cells (Figure 3A). RPPA on samples from a large cohort of newly diagnosed BCP-ALL patients revealed that the level of Vps34 was 9.6-fold higher in BCP-ALL cells compared to healthy bone marrow-derived mononuclear cells (59 BCP-ALL patients vs. 10 healthy controls; $P \leq 0.001$ ) (Online Supplementary Figure S6C). Vps34 levels were also significantly higher in ETV6-RUNX1-positive patient cells in comparison to ETV6-RUNX1-negative BCP ALL (B-Other) patient cells (1.2-fold, $P \leq 0.05)$ (Figure $3 \mathrm{~B})$. In line with this, lower p62 protein levels were observed in ETV6-RUNX1positive in comparison to ETV6-RUNX1-negative BCPALL patient cells (1.4-fold, $P \leq 0.01$ ) (Figure 3B). These RPPA data were confirmed by western blot analysis in a smaller set of patients ( $\mathrm{n}=11$ patients) (Online Supplementary Figure S6A and B).

Next, an siRNA approach was used to investigate whether ETV6-RUNX1 regulates autophagy. To quantify the level of autophagy, the number and volume of LC3B-

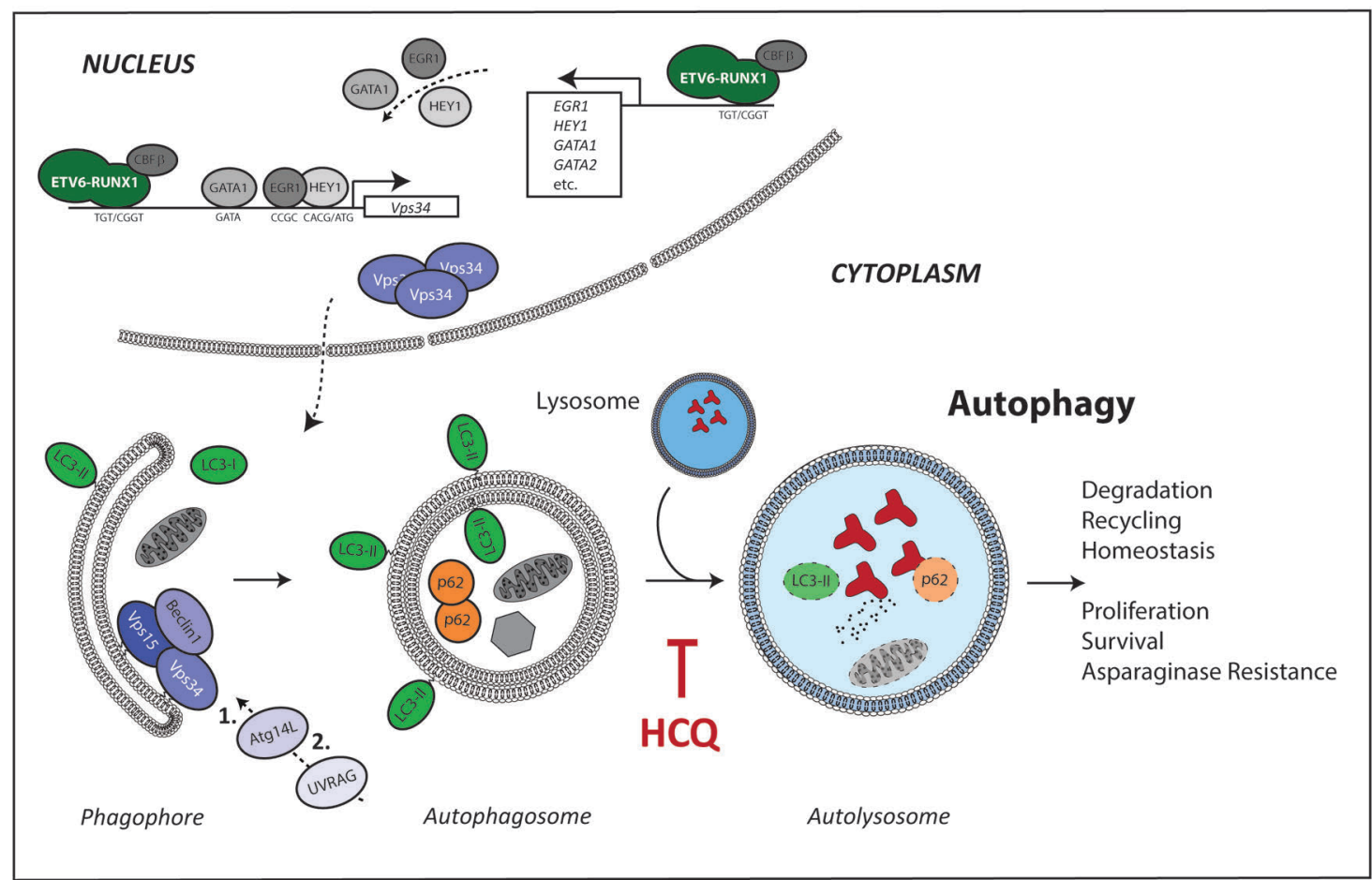

Figure 7. Proposed model for the induction of the Vps34-autophagy pathway in ETV6 RUNX1-positive B-cell precursor acute lymphoblastic leukemia (BCP-ALL) cells. The ETV6-RUNX1 fusion protein can transcriptionally induce the expression of various transcription factors, including GATA1, GATA2, HEY1, and EGR1. ETV6RUNX1 and its co-factor CBF $\beta$, together with GATA1, HEY1 and EGR1 can activate the Vps34 promoter, resulting in enhanced Vps34 expression in ETV6-RUNX1-positive leukemic cells. Vps34, in turn, can initiate autophagy by forming a core autophagy-regulating complex with Beclin 1 and Vps15. This complex plays an important role in: 1) the early initiation (in complex with Atg14L); and 2) the vesicle elongation phase (together with UVRAG) of autophagosome formation. Induction of autophagy allows ETV6-RUNX1-positive cells to maintain homeostasis by degrading and recycling damaged proteins and organelles. In addition, activation of the autophagy program in ETV6-RUNX1-positive cells results in enhanced proliferation, survival and drug resistance. Inhibition of autophagy in ETV6-RUNX1-positive cells, by treatment with hydroxychloroquine $(\mathrm{HCO})$ or knockdown of Vps34, is sufficient to reduce proliferation and survival of leukemic blasts and to induce sensitization to L-Asparaginase. 
positive vesicles were calculated per cell with a $3 \mathrm{D}$ object analyzer using Huygens Professional software (Online Supplementary Figure S6D). Knockdown of ETV6-RUNX1 resulted in a $45 \%$ reduction in the number of $L C 3 B$ positive vesicles and $50 \%$ reduction in the volume of LC3Bpositive vesicles per cell ( $P \leq 0.001$ and $P \leq 0.05$, respectively) (Figure $3 \mathrm{C}-\mathrm{E})$. In addition, knockdown of Vps34 reduced the number of LC3B-positive vesicles and the volume of LC3B-positive vesicles with $79 \%(P \leq 0.001)$ and $84 \%$ $(P \leq 0.05)$, respectively (Figure $3 \mathrm{C}-\mathrm{E})$. These results suggest that autophagy levels are high in ETV6-RUNX1 positive BCP-ALL cells. In addition, both ETV6-RUNX1 and Vps34 are important for maintaining high levels of autophagy in ETV6-RUNX1-positive leukemic cells.

\section{Vps34 is essential for the survival of ETV6-RUNX1-positive leukemic cells}

To determine the functional role of Vps34 in ETV6RUNX1-positive leukemic cells, lentiviral knockdown studies were performed in two ETV6-RUNX1-positive cell lines (REH and REHS1). For these studies, four independent short hairpin RNAs were used. Short hairpins shVps34\#1 and shVps34\#2 were directed against the main Vps34 transcript variants, whereas shVps34\#3 and shVps34\#4 were directed against the two full length transcript variants only (Figure 4A and Online Supplementary Figure S7C). Vps34 knockdown of at least $80 \%$ was achieved with all four individual shRNAs (Figure 4B and C and Online Supplementary Figure S7D). Knockdown of only the full-length Vps34 transcript variants with shVps34\#3 and shVps34\#4 significantly reduced proliferation of the ETV6-RUNX1-positive cell lines REH and REHS1 $(P \leq 0.01)$ (Figure 4A and Online Supplementary Figure S7E and F). Knockdown of all main Vps34 transcript variants with shVps34\#1 and shVps34\#2 resulted in a complete growth arrest of these ETV6-RUNX1-positive cell lines $(P \leq 0.001)$ (Figure 4D and Online Supplementary Figure S7A). In contrast, knockdown of either all main Vps34 transcript variants or the full-length Vps34 transcripts in ETV6-RUNX1negative NALM6 cells decreased proliferation to a significantly lesser extent $(P \leq 0.001)$ (Figure $4 \mathrm{D}$ and Online Supplementary Figure $S 7 A, E$ and F).

To investigate whether the observed growth arrest in ETV6-RUNX1-positive cells was due to a block in cell cycle progression or enhanced apoptosis, flow cytometric analysis was performed using DyeCycle and Annexin V. Cell cycle analysis revealed that knockdown of Vps34 modestly reduces the percentage of cycling ETV6-RUNX1 positive cells (Figure 4E and Online Supplementary Figure $S 7 B$ and $G$ ). In contrast, a remarkable reduction in survival was observed upon Vps34 knockdown. While shRNA mediated knockdown of the full length Vps34 transcript variants in ETV6-RUNX1 positive cells (shRNA\#3 and shRNA\#4) already resulted in 40-50\% apoptotic cells (Online Supplementary Figure S7H), targeting of all main Vps34 transcript variants resulted in even higher levels of apoptosis $(80-90 \%, P<0.01)$ (Figure 4F).

In conclusion, knockdown of Vps34 completely arrests cell growth of ETV6-RUNX1-positive cells by modestly reducing cell cycle progression and strongly inducing apoptosis. Although Vps34 knockdown also affects cell cycle progression and survival in ETV6-RUNX1-negative cells (data not shown), these cells were still able to proliferate.

\section{ETV6-RUNX1-positive ALL cells are relatively sensitive} to hydroxychloroquine

To date, no agents are clinically available that specifically inhibit Vps34 activity. However, the efficacy of autophagy inhibitors is currently being examined in clinical cancer treatment trials. To investigate the effect of autophagy inhibition on ETV6-RUNX1-positive leukemia, we exposed leukemic cells to hydroxychloroquine (HCQ). This agent has favorable pharmacological properties and has been safely used for decades in the treatment of malaria and rheumatoid arthritis. ${ }^{32} \mathrm{HCO}$ accumulates within and de-acidifies the lysosome, resulting in increased LC3B-II levels, which here is indicative of impaired autophagy (Figure 5A). MTT assays were performed to determine the effect of $\mathrm{HCQ}$ on cell viability of ETV6RUNX1-positive and ETV6-RUNX1-negative ALL cell lines (Figure $5 \mathrm{~B}$ and $\mathrm{C}$ and Online Supplementary Figure S8A and $B$ ). While treatment with $20 \mu \mathrm{g} / \mathrm{mL} \mathrm{HCQ}$ resulted in $82 \%$ and $95 \%$ reduced cell viability of ETV6-RUNX1-positive BCP-ALL cell lines (REH and REHS1), the viability of ETV6-RUNX1-negative cell lines was reduced to a lesser extent (NALM6: 43\%, TOM1: 50\%, Loucy: 40\%, Jurkat: $0 \%$ ) (Figure $5 \mathrm{~B}$ ). The IC50 of HCQ was significantly lower in ETV6-RUNX1-positive ALL cell lines compared to ETV6-RUNX1-negative cell lines $(P \leq 0.001)$ (Online Supplementary Figure $S 8 A$ and $B)$. In addition, the effect of $\mathrm{HCQ}$ on the survival of primary BCP-ALL samples was determined. Primary BCP-ALL samples were cultured for five days upon which flow cytometric analysis was performed (see Online Supplementary Figure S8C for flow cytometric gating strategy). Survival of primary ETV6-RUNX1-positive BCP-ALL samples was significantly reduced after treatment with $10 \mu \mathrm{g} / \mathrm{mL} \mathrm{HCQ} \mathrm{(26 \% ,}$ $P \leq 0.05$ ) (Figure 5D and $\mathrm{E}$ ). In contrast, this treatment did not affect cell viability of primary ETV6-RUNX1-negative BCP-ALL samples (Online Supplementary Figure S8D). The survival of primary ETV6-RUNX1-positive ALL samples was even further reduced after treatment with $20 \mu \mathrm{g} / \mathrm{mL}$ $\mathrm{HCQ}(79 \%, P \leq 0.01)$ (Figure $5 \mathrm{E})$. Co-culture of these ALL samples in the presence of primary bone marrow-derived mesenchymal stromal cells (MSCs) significantly rescued the HCQ-mediated induction of apoptosis in primary ETV6-RUNX1-positive ALL samples $(P \leq 0.05)$ (Figure $5 \mathrm{E})$. These results demonstrate that although ETV6-RUNX1positive BCP-ALL cells are relatively sensitive to $\mathrm{HCO}$ treatment, this sensitivity is abrogated by primary MSCs.

\section{Autophagy inhibition sensitizes ETV6-RUNX1-positive acute lymphoblastic leukemia cells to L-Asparaginase}

As our results indicate that inhibition of autophagy reduces survival of ETV6-RUNX1-positive ALL samples, we investigated whether $\mathrm{HCO}$-mediated inhibition of autophagy could sensitize primary BCP-ALL samples to commonly used chemotherapeutics. To investigate the potential of $\mathrm{HCO}$ treatment in sensitization to chemotherapeutics, the percentage of apoptotic cells was determined by flow cytometry after five days of culture either in absence or presence of IC50 values of the chemotherapeutic drug. Our results indicate that $\mathrm{HCQ}$ treatment (in clinically relevant concentrations ${ }^{33,34}$ ) selectively sensitizes ETV6 RUNX1-positive leukemic cells to L-Asparaginase treatment (Figure 6A and Online Supplementary Figure S9A). Treatment of primary ETV6 RUNX1-positive ALL samples with $5 \mu \mathrm{g} / \mathrm{mL}$ or $10 \mu \mathrm{g} / \mathrm{mL} \mathrm{HCQ} \mathrm{resulted} \mathrm{in} \mathrm{a} 48 \%$ 
and $71 \%$ reduction in cell survival during L-Asparaginase exposure, respectively $(P \leq 0.01$ and $P \leq 0.001)$ (Figure $6 \mathrm{~B}$ ), while ETV6-RUNX1-negative BCP-ALL cells were not sensitized to L-Asparaginase (Figure $6 \mathrm{~B}$ and Online Supplementary Figure S9D). Co-culture of primary BCPALL samples with primary MSCs significantly reversed the HCQ-mediated sensitization to L-Asparaginase $(P \leq 0.01)$ (Figure 6C). To investigate whether these primary samples could still be sensitized, similar experiments were performed with a higher dose of $\mathrm{HCQ}(20 \mu \mathrm{g} / \mathrm{mL})$. Inhibition of autophagy with $20 \mu \mathrm{g} / \mathrm{mL} \mathrm{HCQ}$ was indeed sufficient to significantly sensitize primary ETV6-RUNX1positive BCP-ALL samples to L-Asparaginase both in absence or presence of primary MSCs $(80 \%$ reduced survival, $P \leq 0.05$ and $P \leq 0.01$, respectively) (Figure $6 \mathrm{D}$ and Online Supplementary Figure $S 9 B$ and C). In contrast to L-Asparaginase, HCQ did not significantly induce apoptosis of primary BCP-ALL samples upon treatment with prednisolone or 6-mercaptopurine (Online Supplementary Figure S9E and F).

These data show that HCQ-mediated inhibition of autophagy results in sensitization of ETV6-RUNX1-positive BCP-ALL cells, but not ETV6-RUNX1-negative BCPALL cells, to L-Asparaginase.

\section{Discussion}

In this study, we show that the ETV6-RUNX1 fusion gene induces a transcriptional network regulating preleukemic features in hematopoietic progenitors. We show that this network facilitates the induction of autophagy by up-regulating Vps34 expression in ETV6-RUNX1-positive BCP-ALL (Figure 7). In addition, our data show for the first time that inhibition of autophagy is a promising strategy for sensitization of ETV6-RUNX1-positive BCP-ALL cells to the important anti-leukemic agent L-Asparaginase.

The importance of the ETV6-RUNX1 fusion protein for modulation of proliferation, survival and cell cycle distribution has already been shown in cell lines ${ }^{14,17,31}$ and mouse models. ${ }^{10}$ Similarly, expression of the ETV6RUNX1 fusion gene induces survival properties in human cord blood-derived progenitors transplanted in $\mathrm{NOD} / \mathrm{SCID}$ mice or co-cultured in the presence of murine MS-5 stromal cells. ${ }^{11,20}$ However, to date, the downstream effectors of this pro-survival and pro-proliferative phenotype have not been elucidated. In this study, we uncovered the transcriptional network regulating these phenotypes in a human progenitor population by analyzing the gene expression profile after ectopic expression of ETV6RUNX1. This approach allowed us to examine early effects of ETV6-RUNX1 expression in human hematopoietic progenitors. These data, therefore, provide a comprehensive and functional list of ETV6-RUNX1 target genes (Online Supplementary Table S2). In addition to a pro-survival and pro-proliferative phenotype, genes involved in cytoskeleton rearrangements and cellular homeostasis were found to be regulated by the ETV6-RUNX1 fusion protein (Online Supplementary Figure S2). Importantly, our results reveal that autophagy is induced in ETV6-RUNX1positive cells because of transcriptional activation of Vps34, a member of the core (macro)autophagy-regulating complex (Figures 1-3). ${ }^{22}$

In the present study, we show that the ETV6-RUNX1 fusion gene can directly up-regulate the level of autophagy in leukemic cells in absence of cellular stress. Our results demonstrate that these enhanced levels of autophagy are important to maintain proliferation and survival of ETV6RUNX1-positive leukemic cells (Figures 4-6). Knockdown of Vps34 and inhibition of autophagy with $\mathrm{HCQ}$ reduced the proliferation and survival of ETV6 RUNX1-positive BCP-ALL cells, confirming the importance of induced autophagy in these cells (Figures 4 and 5). These results are in line with a recently published study showing sensitization of REH cells to L-Asparaginase during chloroquine treatment in a xenograft model. ${ }^{35}$

Importantly, we found that primary ETV6-RUNX1-negative BCP-ALL samples were not affected by autophagy inhibition. Autophagy might play an important role in protecting leukemic cells during chemotherapeutic treatment with nutrient-modulating drugs like L-Asparaginase that actively inhibits protein biosynthesis by asparagine depletion, which leads to nutritional deprivation and effective killing of leukemic cells. ${ }^{36}$ Here, we show that autophagy selectively protects ETV6-RUNX1-positive leukemic cells against L-Asparaginase treatment, whereas this effect is absent in ETV6-RUNX1-negative leukemic cells (Figure 6). This is in contrast with the study of Takashi et al., ${ }^{35}$ highlighting the importance of the use of primary human leukemic cells in studies investigating targeted therapy. HCQ-mediated inhibition of autophagy did not sensitize cells to prednisolone or 6-mercaptopurine, two other chemotherapeutics often used in treatment of BCP-ALL. These results highlight the importance of the cellular and molecular context in which autophagy inhibition is embedded and show that caution is warranted before the general introduction of autophagy inhibitors in the treatment of leukemia.

The leukemic microenvironment or niche has been shown to protect leukemic cells from elimination by immune responses and chemotherapeutic agents and facilitates the development of drug resistance to classic and targeted chemotherapy. ${ }^{37}$ Here, we show that MSCs can abrogate the effects of autophagy inhibition in ETV6RUNX1-positive BCP-ALL cells. This highlights the crucial role of the leukemic niche in induction of resistance to chemotherapy, including autophagy inhibition. However, MSC-induced resistance of ETV6-RUNX1-positive cells could still be overcome when adequate concentrations of the autophagy inhibitor HCQ were used (Figure 6).

The efficacy of autophagy inhibitors during cancer treatment is currently being examined in clinical trials (reviewed, for example, by White ${ }^{25}$ ). Initial results indicate that $\mathrm{HCQ}$ treatment is safe and tolerated at high concentrations and might be effective in a subset of patients (reviewed, for example, by Vogl et $a$ l. $^{38}$ ). In addition, autophagy independent "off-target" effects of chloroquines, resulting in enhanced response to chemotherapy have been reported. ${ }^{39}$ This strengthens the rationale to use $\mathrm{HCO}$ in clinical practice. However, more specific and potent autophagy inhibitors are currently being developed and preclinical studies with these novel inhibitors (e.g. Lys05) show promising results. ${ }^{40}$ In addition, the recent determination of the crystal structure of Vps34 $4^{41}$ will enable the development of Vps34 inhibitors for clinical use in the near future. ${ }^{42,43}$ Our observation that the ETV6RUNX1 fusion protein induces Vps34 expression and subsequently autophagy, strongly indicates that Vps34/autophagy inhibitors should be considered in future protocols of ETV6-RUNX1-positive BCP-ALL. 


\section{Acknowledgments}

We thank all members of the research laboratory Pediatric Oncology of the Erasmus MC for their help in processing lenkemic and mesenchymal stromal cell samples, in particular E. Bindels and B. de Rooij for scientific input and critical dis- cussions; The Erasmus Optical Imaging Centre for providing support of CLSM; The Department of Hematology of the Erasmus MC for providing the use of CLSM and Flow Cytometers; The Vlietland Ziekenhuis for collecting and providing cord blood.

\section{References}

1. Pui $\mathrm{CH}$, Evans WE. Drug therapy Treatment of acute lymphoblastic leukemia. N Engl J Med. 2006;354(2):166178.

2. Pui $\mathrm{CH}$, Carroll WL, Meshinchi S, Arceci RJ. Biology, risk stratification, and therapy of pediatric acute leukemias: an update. J Clin Oncol. 2011;29(5):551-565.

3. Robison LL. Late effects of acute lymphoblastic leukemia therapy in patients diagnosed at 0-20 years of age. Hematology Am Soc Hematol Educ Program. 2011;2011:238-242.

4. Goldman JM, Melo JV. Chronic myeloid leukemia--advances in biology and new approaches to treatment. N Engl J Med. 2003:349(15):1451-1464

5. Golub TR, Barker GF, Bohlander SK, et al. Fusion of the TEL gene on $12 \mathrm{p} 13$ to the AML1 gene on 21q22 in acute lymphoblastic leukemia. Proc Natl Acad Sci U S A. 1995;92(11):4917-4921.

6. Zelent A, Greaves M, Enver T. Role of the TEL-AML1 fusion gene in the molecular pathogenesis of childhood acute lymphoblastic leukaemia. Oncogene. 2004:23(24):4275-4283

7. Loh ML, Goldwasser MA, Silverman LB, et al. Prospective analysis of TEL/AML1-positive patients treated on Dana-Farber Cancer Institute Consortium Protocol 9501. Blood. 2006;107(11):4508-4513.

8. Stams WA, Beverloo HB, den Boer ML, et al. Incidence of additional genetic changes in the TEL and AML1 genes in DCOG and COALL-treated $\mathrm{t}(12 ; 21)$-positive pediatric ALL, and their relation with drug sensitivity and clinical outcome. Leukemia. 2006;20(3):410-416.

9. van Delft FW, Horsley S, Colman S, et al. Clonal origins of relapse in ETV6-RUNX1 acute lymphoblastic leukemia. Blood. 2011; 117(23):6247-6254

10. Tsuzuki S, Seto $M$, Greaves $M$, Enver $T$. Modeling first-hit functions of the $t(12 ; 21)$ TEL-AML1 translocation in mice. Proc Natl Acad Sci U S A. 2004:101(22):8443-8448.

11. Hong D, Gupta R, Ancliff P, et al. Initiating and cancer-propagating cells in TEL-AML1associated childhood leukemia. Science. 2008;319(5861):336-339.

12. Schindler JW, Van Buren D, Foudi A, et al. TEL-AML1 corrupts hematopoietic stem cells to persist in the bone marrow and initiate leukemia. Cell Stem Cell. 2009. 5(1):43-53.

13. Anderson K, Lutz C, van Delft FW, et al. Genetic variegation of clonal architecture and propagating cells in leukaemia. Nature. 2011;469(7330):356-361

14. Fuka G, Kantner HP, Grausenburger R, et al. Silencing of ETV6/RUNX1 abrogates $\mathrm{PI} 3 \mathrm{~K} / \mathrm{AKT} / \mathrm{mTOR}$ signaling and impairs reconstitution of leukemia in xenografts. Leukemia. 2012;26(5):927-933.

15. Diakos C, Krapf G, Gerner C, et al. RNAimediated silencing of TEL/AML1 reveals a heat-shock protein- and survivin-depen- dent mechanism for survival. Blood. 2007;109(6):2607-2610

16. Mangolini M, de Boer J, WalfVorderwulbecke V, Pieters R, den Boer ML, Williams O. STAT3 mediates oncogenic addiction to TEL-AML1 in t(12;21) acute lymphoblastic leukemia. Blood. 2013; 122(4):542-549.

17. Torrano V, Procter J, Cardus P, Greaves M Ford AM. ETV6-RUNX1 promotes survival of early B lineage progenitor cells via a dysregulated erythropoietin receptor. Blood. 2011:118(18):4910-4918.

18. Gefen N, Binder V, Zaliova M, et al. Hsamir-125b-2 is highly expressed in child hood ETV6/RUNX1 (TEL/AML1) leukemias and confers survival advantage to growth inhibitory signals independent of p53. Leukemia. 2010;24(1):89-96.

19. Palmi C, Fazio G, Savino AM, et al Cytoskeletal Regulatory Gene Expression and Migratory Properties of B Cell Progenitors are Affected by the ETV6RUNX1 Rearrangement. Mol Cancer Res. 2014:12(12):1796-1806.

20. Ford AM, Palmi C, Bueno C, et al. The TELAML1 leukemia fusion gene dysregulates the TGF-beta pathway in early $\mathrm{B}$ lineage progenitor cells. J Clin Invest. 2009; 119(4):826-836

21. Jaber N, Dou Z, Chen JS, et al. Class III PI3K Vps34 plays an essential role in autophagy and in heart and liver function. Proc Natl Acad Sci U S A. 2012:109(6):2003-2008.

22. Funderburk SF, Wang QJ, Yue Z. The Beclin 1-VPS34 complex--at the crossroads of autophagy and beyond. Trends Cell Biol. 2010;20(6):355-362.

23. Levine B, Klionsky DJ. Development by self-digestion: molecular mechanisms and biological functions of autophagy. Dev Cell. 2004;6(4):463-477.

24. Levine B, Kroemer G. Autophagy in the pathogenesis of disease. Cell. 2008; 132(1):27-42.

25. White E. Deconvoluting the contextdependent role for autophagy in cancer. Nat Rev Cancer. 2012;12(6):401-410.

26. Yeoh EJ, Ross ME, Shurtleff $S A$, et al Classification, subtype discovery, and prediction of outcome in pediatric acute lymphoblastic leukemia by gene expression profiling. Cancer Cell. 2002;1(2):133-143.

27. Gandemer V, Rio AG, de Tayrac M, et al. Five distinct biological processes and 14 differentially expressed genes characterize TEL/AML1-positive leukemia. BMC Genomics. 2007:8:385

28. Andersson A, Olofsson T, Lindgren D, et al Molecular signatures in childhood acute leukemia and their correlations to expression patterns in normal hematopoietic subpopulations. Proc Natl Acad Sci U S A. 2005:102(52):19069-19074.

29. Fine BM, Stanulla M, Schrappe M, et al. Gene expression patterns associated with recurrent chromosomal translocations in acute lymphoblastic leukemia. Blood. 2004; 103(3): 1043-1049.

30. van $\operatorname{der}$ Veer A, Waanders E, Pieters R, et al
Independent prognostic value of $\mathrm{BCR}$ ABL1-like signature and IKZF1 deletion, but not high CRLF2 expression, in children with B-cell precursor ALL. Blood. 2013, 122(15):2622-2629

31. Fuka G, Kauer M, Kofler R, Haas OA Panzer-Grumayer R. The leukemia-specific fusion gene ETV6/RUNX1 perturbs distinct key biological functions primarily by gene repression. PLoS One. 2011;6(10):e26348.

32. Mackenzie AH. Antimalarial drugs for rheumatoid arthritis. Am J Med. 1983;75(6A):48-58

33. Munster T, Gibbs JP, Shen D, Baethge BA, Botstein GR, Caldwell J, et al Hydroxychloroquine concentrationresponse relationships in patients with rheumatoid arthritis. Arthritis Rheum. 2002;46(6):1460-1469.

34. Rangwala R, Leone R, Chang YC, et al. Phase I trial of hydroxychloroquine with dose-intense temozolomide in patients with advanced solid tumors and melanoma. Autophagy. 2014;10(8):13691379

35. Takahashi H, Inoue J, Sakaguchi K, Takagi $\mathrm{M}$, Mizutani S, Inazawa J. Autophagy is required for cell survival under L-asparaginase-induced metabolic stress in acute lymphoblastic leukemia cells. Oncogene. 2017; 36(30):4267-4276

36. Pieters R, Hunger SP, Boos J, et al. Lasparaginase treatment in acute lymphoblastic leukemia: a focus on Erwinia asparaginase. Cancer. 2011;117(2):238-249.

37. McMillin DW, Negri JM, Mitsiades CS. The role of tumour-stromal interactions in modifying drug response: challenges and opportunities. Nat Rev Drug Discov. 2013; 12(3):217-228.

38. Vogl DT, Stadtmauer EA, Tan KS, et al. Combined autophagy and proteasome inhibition: A phase 1 trial of hydroxychloroquine and bortezomib in patients with relapsed/refractory myeloma. Autophagy. 2014;10(8):1380-1390.

39. Maes H, Kuchnio A, Peric A, et al. Tumor Vessel Normalization by Chloroquine Independent of Autophagy. Cancer Cell. 2014;26(2):190-206

40. McAfee Q, Zhang Z, Samanta A, et al Autophagy inhibitor Lys05 has single-agent antitumor activity and reproduces the phenotype of a genetic autophagy deficiency. Proc Natl Acad Sci U S A. 2012; 109(21):8253-8258

41. Miller S, Tavshanjian B, Oleksy A, et al Shaping development of autophagy inhibitors with the structure of the lipid kinase Vps34. Science. 2010;327(5973): 1638-1642.

42. Ronan B, Flamand O, Vescovi L, et al. A highly potent and selective Vps34 inhibitor alters vesicle trafficking and autophagy. Nat Chem Biol. 2014;10(12):1013-1019.

43. Dowdle WE, Nyfeler B, Nagel J, et al Selective VPS34 inhibitor blocks autophagy and uncovers a role for NCOA4 in ferritin degradation and iron homeostasis in vivo. Nat Cell Biol. 2014;16(11):1069-1079. 